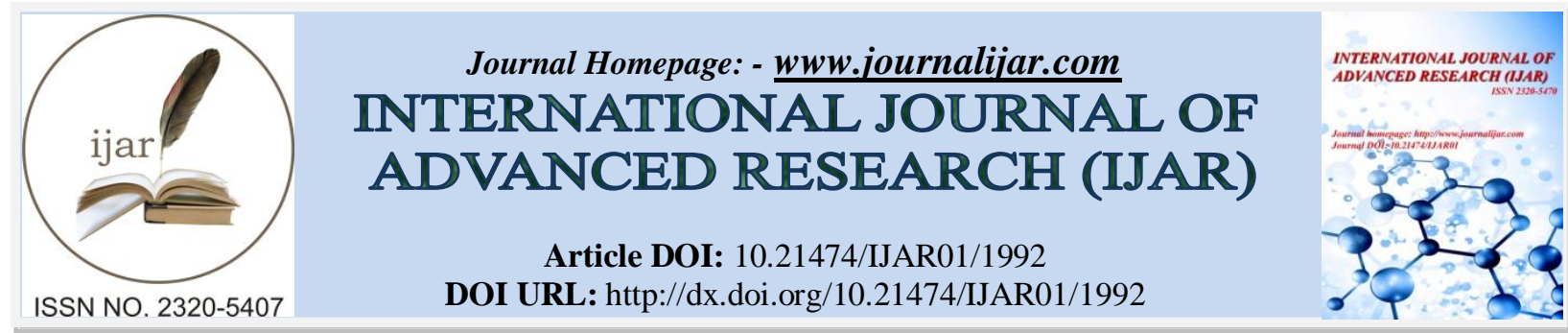

RESEARCH ARTICLE

\title{
TOWARD AN INNOVATIVE AND NEW METHODOLOGICAL DEMARCHE ON MASTER'S THESIS MANAGEMENT.
}

KHALID ASSALAOU.

Department of Physics, Abdelmalek Essaâdi University, Polydisciplinary Faculty of Larache, Morocco.

\section{Manuscript Info}

Manuscript History

Received: 12 August 2016

Final Accepted: 22 September 2016

Published: October 2016

Key words:-

Diagnostic

Pré-acquired / Prerequisites,

Synergy

Immersion

Formation-Action

Formative Evaluation

\begin{abstract}
In order to ensure a good fundamental involvement of the candidates for licenses, in particular, in the environmental, economical and relational concerns, that are universally proven, we have adopted a mode of adult education management based on principles of self and assisted co-formation, advocating the values of empowerment and synergy through immersion techniques in individual, collective and groupal projects. The DOREAS (Development and Optimization of Renewable Energy on Agricultural System) project aims to create a means of motivation and positive outcome generated by the formationaction and the guidance, so as to make the end-of-study projects in the physics field more significant. Indeed, the students worked in pairs with differentiated and complementary tasks to design, prepare and implement each element of a development and optimization mechanism for an agricultural system based on renewable energy. After the analysis step which is supposed to determine the deviation of the preacquired by reporting the prerequisites, the purpose of the preoperative phase has been to fill the identified gaps, through a training session based on the principles of guidance. This has allowed the development of the equipments and the software knowledge's which are deemed very important to realize the project. Then, the latter has been materialized on a functional model of ecological farm where photovoltaic pumping, irrigation, lighting, air conditioning of the greenhouse and the phytosanitary local security are automatically triggered.

Various assessments have finally resulted many successes in skills, abilities, skills of students, adult education approaches, social and economical benefits and have provided access to many perspectives of multidisciplinary extensions.
\end{abstract}

Copy Right, IJAR, 2016,. All rights reserved.

\section{Introduction:-}

Un feedback sur les projets de recherche habituellement menés au sein des établissements universitaires laisse entendre que, pour les nouvelles technologies en particulier, l'encadrement se base sur des procédés encore entachées de dogmatisme et bornées à la phase de simulation. Ces projets souffrent en général de défaut de liens avec les problèmes vécus au quotidien par l'étudiant et son environnement social, de manque de rapport direct avec les politiques et les programmes établis dans le cadre des finalités étatiques si ce n'est des projets sociétaux, 
d'ignorance des pré-requis / pré-acquis (Vieillevoye et al., 2012) devant être pris en compte en amont de toute conception de thématique de recherche, d'absence de la notion de synergie (Meirieu, 1996) dans l'attribution des tâches (de Montmollin, 1984) de recherches et de non aboutissement à des réalisations / innovations concrètes sur terrain.

L'étudiant-chercheur est de ce fait appelé à exécuter bon gré mal gré si ce n'est machinalement des séries d'opérations focalisées rien que sur l'obtention d'une attestation ou d'un diplôme. Son but réside donc dans sa qualification plutôt que dans son utilité pour la société si ce n'est pour l'univers. Un tel processus instaure en fait un climat de dépaysement qui atténue l'engagement, faute de démarches valorisantes d'apprentissage susceptibles de lier l'académique à l'empirique, l'utile à l'agréable, à la lumière des principes d'autonomisation (Pillonel, 2001), de motivation et d'implication (Charles, 2012) chaleureusement requis dans les pédagogies nouvelles et des impératifs de recherche, traitement et investissement requis dans les stratégies de développement.

Le projet DOSAER (Développement et Optimisation d'un Système Agricole à Energies Renouvelables) est né dans cette optique d'amélioration des tenants et des aboutissants de la formation tout en s'inspirant des orientations écologiques marocaines telles le programme " NOOR" de l'énergie solaire, le plan Maroc Vert (PMV), la stratégie agricole nouvelle mise en place par le Ministère de l'Agriculture et des Pêches Maritimes, la Stratégie Nationale de l'Eau, le Programme de Pompage Solaire et, en particulier, le projet COP22 inhérent à la protection de l'environnement.

Notre inspiration-ci a mûri lors de quelques visites de fermes aux environs de Larache, ville chef lieu du Loukous, région du Nord, une plaine connue en tant que lieu privilégié par rapport aux autres plaines du Maroc de par sa pluviosité, sa nature de sol et la variété de sa productivité agricole.

Il s'est souvent agi de fermes assez bien aménagées qui se présentaient sous forme de terrains découverts et / ou semés de serres, assez bien alimentées en eau à partir de puits munis de pompes immergées qui remplissaient des bassins dotés à leur tour de pompes surfaciques. L'alimentation des pompes y était toujours assurée par le réseau électrique qui occasionnait malheureusement des dépenses budgétaires assez importantes ou par recours au butane si ce n'est au gasoil, deux sources d'énergies dont la nocivité s'ajoutait à celle de l'usage des produits phytosanitaires.

De telles visites poussent à s'interroger sur les inconvénients de ces utilisations énergétiques aussi bien que sur des alternatives à la lumière des nouvelles orientations universelles en matière d'énergie propre.

D'autres questions s'imposent dont l'utilisation irrationnelle de l'eau dans la mesure où l'irrigation se fait sans soucis d'économie et sans dosage ni quantitatif ni temporel.

L’idée de recours à l'énergie photovoltaïque surgit alors comme moyen de réaliser des gains pécuniers tout en respectant l'environnement : plus de factures d'électricité pour le cultivateur et plus de pollution ni pour la faune ni pour la flore.

Quant au gaspillage de l'eau, il a fallu trouver des moyens à même de doser l'usage de cette matière en fonction des besoins spécifiques des plantes et selon une cadence dictée automatiquement par le besoin du sol.

En parallèle, les risques des produits phytosanitaires nécessaires sauraient trouver des moyens d'évitement par une sécurisation des lieux de leur stockage.

Un projet se dessine alors à l’horizon. Il se présente comme suit : 


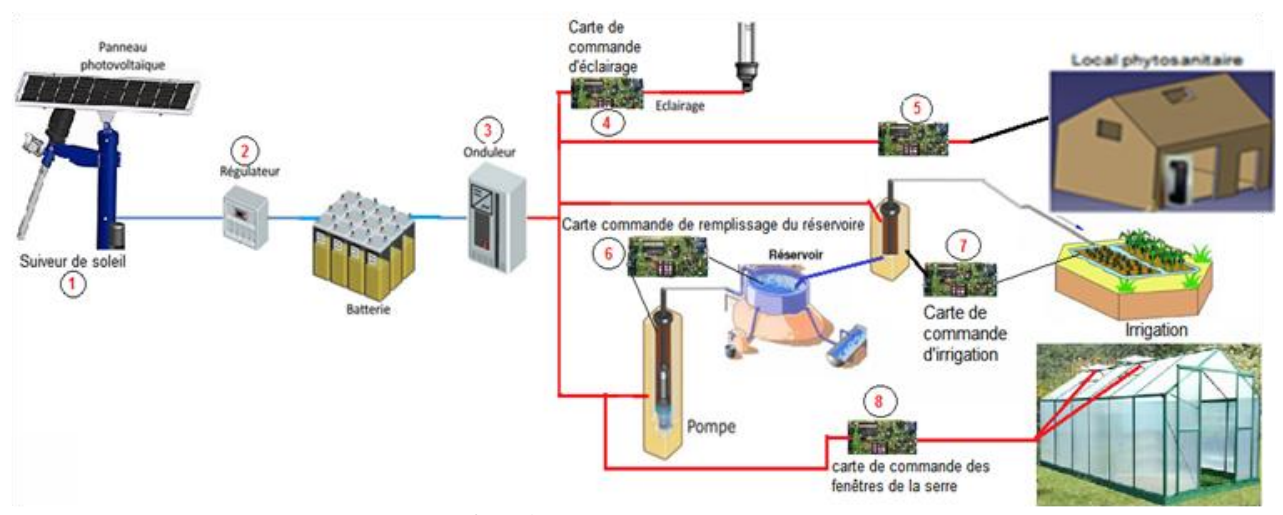

Fig. 1. Conception du projet DOSAER

Il est là une possibilité de montage de panneaux solaires photovoltaïques sur un suiveur de soleil, montage qui servira à optimiser la production photovoltaïque (1) et à produire l'énergie électrique sous forme de courant continu stocké dans des batteries à travers un régulateur (2). Ce dernier permettra de réguler le niveau d'énergie stockée par les batteries dont il empêchera les surcharges ou les décharges trop profondes. Quant à l'onduleur (3), il aura pour fonction de convertir le courant continu fourni par les batteries en courant alternatif afin d'alimenter la ferme. Celleci est composée d'un système d'éclairage (4), d'un système de surveillance du local de stockage des produits phytosanitaire (5), d'un système de remplissage de réservoir (6), d'un système de commande d'irrigation (7) et d'un système automatique de régulation de la température dans une serre photovoltaïque (8).

Parallèlement, l'idée est venue d'intégrer (Rogiers, 2001) les étudiants à la conception et la réalisation du prototype envisagé avec une visée d'amélioration de leur formation à caractère souvent dogmatique en matière d'apprentissage de l'électronique et d'adoption d'approches innovantes, différentielles, communicatives, participatives, interactive et contractuelles (Tardif, 2005)(Kahn, 2010)(Baylon and Mignot, 1994), des approches par projets (Proulx, 2008)( Galand and Frenay, 2005], par compétences et par capacités, approches fondées plutôt sur des méthodes actives d'immersion qui mettent en jeu les principes de motivation, d'implication et d'autonomisation accordées à la personne et aux groupes (Presse et al., 2010).

Aussi les étudiants auraient-ils la chance de se former dans le cadre de «recherches-actions » (Proulx, 2008) et partant, la formation se convertirait en «form'action», là où la science trouverait une significativité dans la manipulation du réel, la résolution des problèmes du vécu, la mise en jeu des capacités personnelles et synergiques, l'entrainement à l'extrapolation et à la conceptualisation, la mise en commun et la négociation des résultats pour accéder enfin à un produit collectif, palpable et utile, une irrigation saine, automatique et bon marché en l'occurrence, ce produit même qui servirait d'objet du projet de fin d'études et de moyen d'évaluation des compétences. Le projet du professeur et / ou de l'institution se verrait donc heureux de la complicité des étudiants dans une démarche andragogique (Bourgeois and Nizet, 1997).

Aussi avons-nous lancé le projet DOSAER au sein de la faculté.

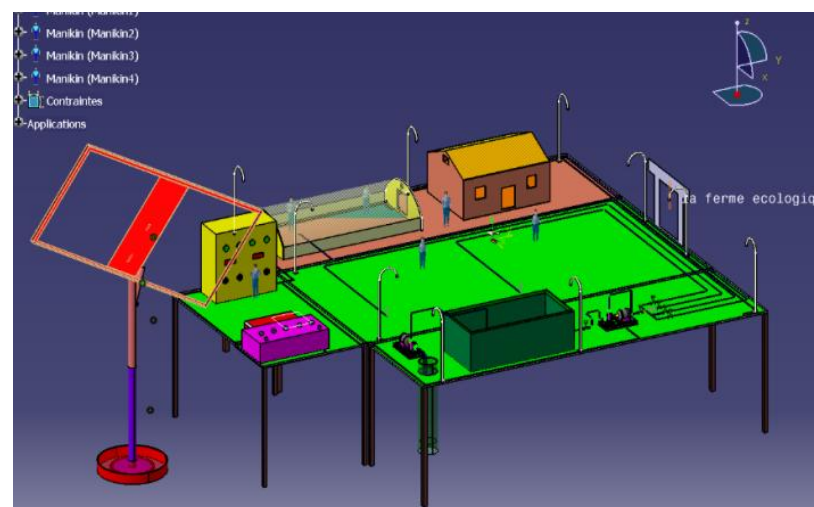

Fig. 2. Maquette du système Agricole à énergie renouvelable 


\section{MATERIEL ET METHODES :-}

Sur le plan méthodologique, nous avons opté pour une "form'action" dont l'organisation se présente toujours dans un esprit d'immersion des apprenants et de responsabilisations individuelles et groupales en vue d'initier à l'inventivité à partir du brassage des pré-acquis, ces derniers devant constituer une matière d'investissement et un tremplin de développement de la personnalité.

Notre initiative se présente en deux phases :

- une phase préopératoire de diagnostic, de consolidation et d'appui aux aptitudes requises pour l'entreprise dudit projet

- et une phase opératoire de production en autonomie assistée menée dans un esprit de guidance et sous l'observance des techniques des APARs (Ateliers de Production, d'Approfondissement et de Régulation) et des mises en commun qui mettent en exergue la valeur de synergie.

Pour ce qui est du matériel, nous en ferons une description implicite à travers les phases susmentionnées.

\section{LA PHASE PREPARATOIRE DE LA FORMATION :-}

Il s'agit là d'une phase préparatoire déclenchée par un diagnostic, lequel diagnostic s'avise une délimitation des pré-requis et une vérification suffisante des pré-acquis en vue d'asseoir convenablement les consolidations nécessaires, combler les lacunes et faciliter l'intégration.

De ce diagnostic, un certain nombre de connaissances se sont avérées étrangères au cursus normal poursuivi à la faculté, ce qui nous a poussés à programmer des formations préparatoires menées en petits groupes à tâches uniques.

A l'issue de ces activités menées en autonomie, chaque atelier était en plénière appelé à rendre compte de ses algorithmes et résultats puis l'ensemble des ateliers, à confronter et négocier les différents apports dans le dessein d'en sélectionner le meilleur processus de raisonnement exposé, le plus rapide et le plus efficace, d'affiner ce processus pour en faire une procédure à mémoriser par recours à d'autres situations équivalentes. Il en est là un pas vers les stratégies de modélisation générées par un coaching imperceptible mais efficient.

Aussi les étudiants ont-ils pu s'imprégner de la notion d'énergie renouvelable, des techniques de programmation des circuits intégrés (PIC) et des cartes ARDUINO, du mode d'emploi des logiciels MPLAB de la programmation des PICs, PSIM, ISIS, CATIA, LABVIEW et PVSYST respectivement faits pour la modélisation des panneaux solaires, pour la simulation des circuits électroniques , pour la conception / simulation / design, pour la supervision et pour le dimensionnement des panneaux photovoltaïques.

\section{STRUCTURATION DE LA FORMATION PREOPERATOIRE :-}

Cette phase préparatoire a d'autre part été renforcée par le recours à des personnes ressources dont un éminent ingénieur agronome et propriétaire foncier qui, au sein même de sa ferme, a pu élucider certains éléments d'informations inhérents au dosage des plantes en fonction de leurs besoins, et un autre ingénieur qui a su documenter les étudiants en matière de dimensionnement des panneaux photovoltaïques et de pompes solaires.

Ces initiations ont bel et bien permis aux étudiants de s'approprier une terminologie, un certain nombre de concepts et techniques jugés nécessaires à l'entreprise du projet en question, le projet DOSAER dont ils ont tout de suite été impliqués en matière de conceptualisation, de planification, de mise en œuvre et d'évaluation. 
Table 1 :- Programme de formation préopératoire

\begin{tabular}{|c|c|c|c|c|}
\hline Thématiques & $\begin{array}{c}\text { Situations et supports } \\
\text { selon les séances }\end{array}$ & $\begin{array}{l}\text { Consignes de } \\
\text { recherché }\end{array}$ & $\begin{array}{l}\text { Durées fois } \\
\text { séances }\end{array}$ & Produits attendus \\
\hline Notion d'énergie solaire & $\begin{array}{l}\text { Image présentant les } \\
\text { différents types } \\
\text { d'énergies } \\
\text { renouvelables } \\
\text { articles variés traitant } \\
\text { la question d'énergie }\end{array}$ & $\begin{array}{l}\text { Dresser une table de } \\
\text { spécification des } \\
\text { énergies (types, } \\
\text { caractéristiques, } \\
\text { usage) }\end{array}$ & $\begin{array}{l}30 ’ x 2 \text { en s/s } \\
\text { groupes } \\
70 ’ x 2 \text { en } \\
\text { plénière }\end{array}$ & $\begin{array}{l}\text { Table de } \\
\text { spécification }\end{array}$ \\
\hline $\begin{array}{l}\text { I. Composantes d'une } \\
\text { installation PV }\end{array}$ & $\begin{array}{l}\text { 1. Schéma renfermant } \\
\text { les différentes } \\
\text { composantes } \\
\text { 2. Articles décrivant } \\
\text { chaque composante }\end{array}$ & $\begin{array}{l}\text { - Elaborer une } \\
\text { typologie des } \\
\text { composantes, de } \\
\text { leurs } \\
\text { caractéristiques et } \\
\text { de leurs modes de } \\
\text { fonctionnement } \\
\end{array}$ & $\begin{array}{l}\text { - 40’ x } 2 \text { en } \\
\text { s/s } \\
\text { groupes } \\
\text { - 90' en } \\
\text { plénière }\end{array}$ & $\begin{array}{l}\text { Une fiche } \\
\text { signalétique pour } \\
\text { chaque composante }\end{array}$ \\
\hline $\begin{array}{ll}\text { II. } & \text { Exploration du } \\
\text { logiciel PSIM }\end{array}$ & $\begin{array}{l}\text { 3. Guide d'utilisation } \\
\text { du logiciel } \\
\text { 4. Exercices } \\
\text { d'application }\end{array}$ & $\begin{array}{l}\text { - explorer } \\
\text { collectivement le } \\
\text { logiciel } \\
\text { - Sur PC, simuler une } \\
\text { activité d'usage du } \\
\text { logiciel }\end{array}$ & $\begin{array}{l}\text { - } 30 \text { ’ x } 3 \text { en } \\
\text { s/s } \\
\text { groupes } \\
\text { - 70’ x } 3 \text { en } \\
\text { plénière }\end{array}$ & $\begin{array}{l}\text { Modèle } \\
\text { d'application du } \\
\text { logiciel }\end{array}$ \\
\hline $\begin{array}{ll}\text { II. } & \text { Dimensionnement et } \\
\text { installation des } \\
\text { panneaux } \\
\text { photovoltaïques }\end{array}$ & $\begin{array}{l}\text { 1. Articles variés et } \\
\text { illustrés portant sur } \\
\text { le dimensionnement } \\
\text { et les installations } \\
\text { 2. Etude de cas d'une } \\
\text { installation de } \\
\text { pompage solaire }\end{array}$ & $\begin{array}{l}\text { - Relever les étapes } \\
\text { de } \\
\text { dimensionnement } \\
\text { de chaque situation } \\
\text { présentée par les } \\
\text { articles distribués } \\
\text { - imaginer le } \\
\text { dimensionnement } \\
\text { de l'installation } \\
\text { proposée }\end{array}$ & $\begin{array}{l}\text { - } 60 \text { ’ x } 2 \text { en } \\
\text { s/s } \\
\text { groupes } \\
\text { - } 120^{\prime} \text { x } 2 \\
\text { en plénière }\end{array}$ & $\begin{array}{l}\text { 1. Tableau } \\
\text { synoptique des } \\
\text { différentes } \\
\text { installations PV } \\
2 . \quad \text { Tableau } \\
\text { récapitulatif des } \\
\text { différentes } \\
\text { composantes du } \\
\text { cas proposé }\end{array}$ \\
\hline IV. Les capteurs & $\begin{array}{l}\text { 1. Image présentant } \\
\text { les différents types } \\
\text { de capteurs } \\
\text { 2. Articles variés } \\
\text { traitant l'étalonnage } \\
\text { des capteurs }\end{array}$ & $\begin{array}{l}\text { Dresser une table } \\
\text { de spécification des } \\
\text { capteurs (types, } \\
\text { caractéristiques, } \\
\text { usage) }\end{array}$ & $\begin{array}{l}\text { - 30’' x } 2 \text { en } \\
\text { s/s } \\
\text { groupes } \\
\text { - 70’ x } 2 \text { en } \\
\text { plénière }\end{array}$ & $\begin{array}{l}\text { Table de } \\
\text { spécification }\end{array}$ \\
\hline \begin{tabular}{|l} 
V. \\
Notion de \\
programmation des \\
circuits intégrés \\
(PIC)
\end{tabular} & $\begin{array}{l}\text { 1. documents sur les } \\
\text { différents types de } \\
\text { programmation } \\
\text { 2. articles variés } \\
\text { traitant de la } \\
\text { programmation des } \\
\text { PIC }\end{array}$ & $\begin{array}{l}\text { - Dresser une } \\
\text { typologie }\end{array}$ & $\begin{array}{l}\text { - } 30 \text { ’x } 3 \text { en } \\
\text { s/s } \\
\text { groupes } \\
\text { - 70’x } 3 \text { en } \\
\text { plénière }\end{array}$ & $\begin{array}{l}\text { Table de } \\
\text { spécification }\end{array}$ \\
\hline $\begin{array}{l}\text { VI. Découverte / } \\
\text { utilisation du logiciel } \\
\text { ISIS }\end{array}$ & $\begin{array}{l}\text { 1. Guide d'utilisation } \\
\text { du logiciel } \\
\text { 2. Exercices } \\
\text { d'application }\end{array}$ & $\begin{array}{l}\text { - explorer } \\
\text { collectivement le } \\
\text { logiciel } \\
\text { - Sur PC, simuler une } \\
\text { activité d'usage du } \\
\text { logiciel } \\
\text { - Transposer la } \\
\text { simulation en } \\
\text { réalisations } \\
\text { pratiques } \\
\end{array}$ & $\begin{array}{l}\text { - } 30 \text { ’ x } 4 \text { en } \\
\text { s/s } \\
\text { groupes } \\
\text { 70’ x } 4 \text { en } \\
\text { plénière }\end{array}$ & $\begin{array}{l}\text { Processus de } \\
\text { fonctionnement } \\
\text { réussi }\end{array}$ \\
\hline
\end{tabular}




\begin{tabular}{|c|c|c|c|c|c|}
\hline & $\begin{array}{l}\text { Découverte de la } \\
\text { Plate-forme } \\
\text { ARDUINO et } \\
\text { entrainement sur son } \\
\text { utilisation }\end{array}$ & $\begin{array}{l}\text { 1. Carte Arduino } \\
\text { isolée (fig. } 3 \text { ) } \\
\text { 2. Guide d'utilisation } \\
\text { de la carte } \\
\text { 3. Exercices pratiques } \\
\text { d'utilisation de la } \\
\text { carte }\end{array}$ & $\begin{array}{l}\text { - Sur PC, simuler une } \\
\text { activité d'usage de } \\
\text { la carte sous le } \\
\text { logiciel Isis } \\
\text { - Transposer la } \\
\text { simulation en } \\
\text { réalisations } \\
\text { pratiques }\end{array}$ & $\begin{array}{l}\text { - } 30 \text { ' x } 4 \text { en } \\
\text { s/s } \\
\text { groupes } \\
\text { 70’’x } 4 \text { en } \\
\text { plénière }\end{array}$ & $\begin{array}{l}\text { Processus de } \\
\text { fonctionnement } \\
\text { réussi }\end{array}$ \\
\hline & $\begin{array}{l}\text { Mode d'emploi du } \\
\text { logiciel CATIA }\end{array}$ & $\begin{array}{l}\text { 1. Guide d'utilisation } \\
\text { du logiciel } \\
\text { 2. Exercices } \\
\text { d'application du } \\
\text { logiciel. }\end{array}$ & $\begin{array}{l}\text { - Dessiner une à une } \\
\text { les composantes de } \\
\text { la maquette en } \\
\text { binôme } \\
\text { - Présenter les } \\
\text { productions aux } \\
\text { autres binômes } \\
\text { - Négocier les } \\
\text { différents apports } \\
\text { en plénières en vue } \\
\text { d'établir un } \\
\text { prototype }\end{array}$ & $\begin{array}{l}\text { - } 30 \text { ’ x 4en } \\
\text { s/s } \\
\text { groupes } \\
\text { - 70’ x } 4 \text { en } \\
\text { plénière }\end{array}$ & $\begin{array}{l}\text { Prototype construit } \\
\text { synergiquement }\end{array}$ \\
\hline
\end{tabular}

UNE PHASE OPERATOIRE DE PRODUCTION :-

STRUCTURATION DE LA FORMATION PREOPERATOIRE :-

Or, étant donné la lourdeur de la tâche, DOSAER a dû se ramifier en sous-projets distribués à des ateliers à tâches différentes et convergentes, soit sept ateliers de deux personnes chacun. La répartition des tâches se présente comme suit:

Table 2 :- Programme de formation opératoire de production

\begin{tabular}{|c|c|c|c|c|c|}
\hline 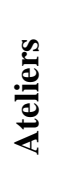 & Conceptions \& réalisations visées & 言 & 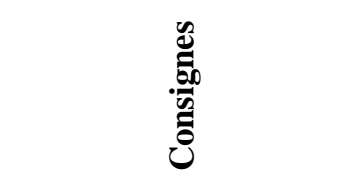 & 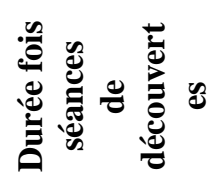 & 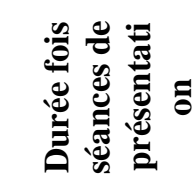 \\
\hline 1 & Un Suiveur de Soleil Bi-Axial & \multirow{7}{*}{ 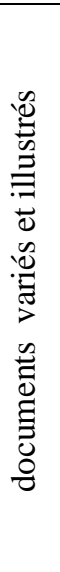 } & \multirow{7}{*}{$\begin{array}{l}\text { - dessiner le plan de la } \\
\text { composante demandée } \\
\text { - simuler cette } \\
\text { conception sur PC } \\
\text { - produire une carte de } \\
\text { commande } \\
\text { - Elaborer une maquette }\end{array}$} & \multirow{7}{*}{$\begin{array}{l}120^{\prime} \text { x } 4 \\
\text { séances / } \\
\text { semaine } \\
\text { (17 semaines) }\end{array}$} & \multirow{7}{*}{$\begin{array}{l}120 ' \times 1 / \\
\text { semaine }(17 \\
\text { semaines })\end{array}$} \\
\hline 2 & $\begin{array}{l}\text { Un régulateur/chargeur de batterie au } \\
\text { plomb } 12 \mathrm{v} / 12 \mathrm{Ah}\end{array}$ & & & & \\
\hline 3 & Un onduleur solaire & & & & \\
\hline 4 & $\begin{array}{l}\text { Une carte de commande d'une station } \\
\text { de pompage d'eau }\end{array}$ & & & & \\
\hline 5 & $\begin{array}{l}\text { Une carte de commande d'irrigation } \\
\text { automatique }\end{array}$ & & & & \\
\hline 6 & $\begin{array}{l}\text { Le système de régulation de la } \\
\text { température dans une serre } \\
\text { Photovoltaïque }\end{array}$ & & & & \\
\hline 7 & $\begin{array}{l}\text { L'éclairage / surveillance } \\
\text { automatiques du local de stockage } \\
\text { des produits phytosanitaires }\end{array}$ & & & & \\
\hline
\end{tabular}

\section{LA CARTE ARDOUINO :-}

En amont, tous les ateliers qui ont agi en vue d'élaborer notre maquette ont opté pour la carte Arduino qui présente des avantages étant donné :

- $\quad$ son caractère bon marché,

- la simplicité et la clarté de son environnement de programmation,

- la multiplicité de ses plateformes (adaptable pour Windows, Macintosh et Linux),

- la disponibilité de ses librairies et la diversité de ses fonctions implémentées,

- l'accessibilité inconditionnelle de son logiciel open source, 
- la présence permanente en ligne des tutoriaux et exemples y afférents (forums, site perso etc...)

- l'existence de « Shield», boucliers qui se présentent sous forme de cartes supplémentaires (afficheur graphique couleur, interface Ethernet, GPS, etc.)connectables sur le module Arduino.

Il s'agit en effet d'une plateforme open-source d'électronique basée sur une simple carte à microcontrôleur autours d'un microcontrôleur ATmega2560 et de composantes complémentaires qui facilitent la programmation et l'interfaçage avec d'autres circuits.

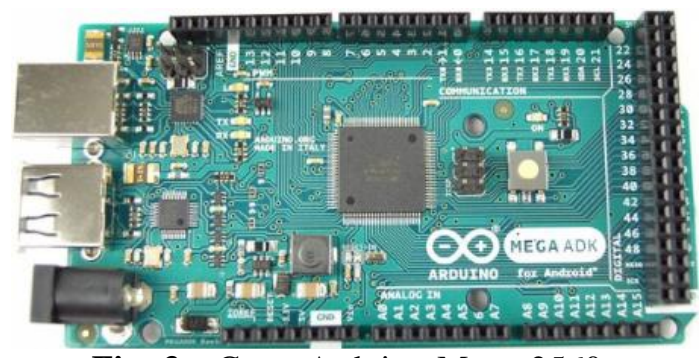

Fig. 3. Carte Arduino Mega 2560

Le microcontrôleur est préprogrammé avec un « bootloader » de façon à ce qu'un programmateur dédié ne soit pas nécessaire. La carte Mega2560 peut être programmée avec le logiciel Arduino qui permet d'écrire, compiler et transférer le programme vers la carte à microcontrôleur. Il suffit simplement de la connecter à un ordinateur à l'aide d'un câble USB et de sélectionner "Arduino Mega" dans le menu Tools > Board.

La carte Arduino Mega dispose 54 broches numériques d'entrée / sortie (dont 14 peuvent être utilisés comme sorties PWM), 16 broches d'entrées/sorties analogiques (qui peuvent également être utilisées en broches entrées/sorties numériques), un quartz $16 \mathrm{MHz}$, d'un connecteur d'alimentation jack, d'un connecteur ICSP (programmation "incircuit"), une connexion USB, et d'un bouton de réinitialisation. Une vue réelle de la carte est présentée dans la figure $_{2}$.

En outre, cette carte peut être alimentée via la connexion USB (qui fournit $5 \mathrm{~V}$ jusqu'à $500 \mathrm{~mA}$ ) ou avec une alimentation externe qui fournit une tension recommandée entre $7 \mathrm{~V}$ et $12 \mathrm{~V}$ et qui peut provenir de la batterie ou d'un adaptateur AC -DC. La source d'alimentation est automatiquement sélectionnée par la carte.

Conformément au planning établi pour la form'action, nous soumettons ci-après les apports des ateliers de conception / réalisations des composantes de notre maquette.

\section{RESULTATS :-}

DES APPORTS D'ATELIERS EN FORM'ACTION :Atelier1 : Le suiveur de soleil bi-axial

Il s'agit du produit de l'atelier ${ }_{1}$ qui s'est intéressé à la mise en œuvre d'un prototype de suiveur pour la trajectoire solaire, prototype distinct par son activité automatique et permanente le long de la journée et par son rendement élevé eût égard aux panneaux fixes. Dans un premier temps, les étudiants ont pu détailler un volet mécanique où ils ont étudié et fabriqué deux types de mouvements, rotatif et linéaire, par l'intermédiaire de deux moteurs à courant continu. Dans un deuxième temps, ils se sont consacrés au volet électronique où ils ont élaboré une programmation de la carte Arduino. Dans un troisième temps, à l'aide du logiciel Proteus qui intègre ISIS, ils ont pu procéder à une simulation de la carte en question dont la réussite les a conduits à la confirmation de l'efficacité du programme. Le quatrième temps a été consacré à la réalisation d'une carte de commande.

La valeur ajoutée par notre recherche-action réside en :

- la rationalisation des choix de moteurs à base de calculs théoriques de haute précision et par lesquels la masse de la structure mécanique est devenue supportable,

- la conception d'un nouveau modèle de suiveur qui se distingue par un mécanisme moins ardu,

- la facilitation de la programmation par le truchement de la carte ARDUINO plutôt que par les autres microcontrôleurs (PIC). 

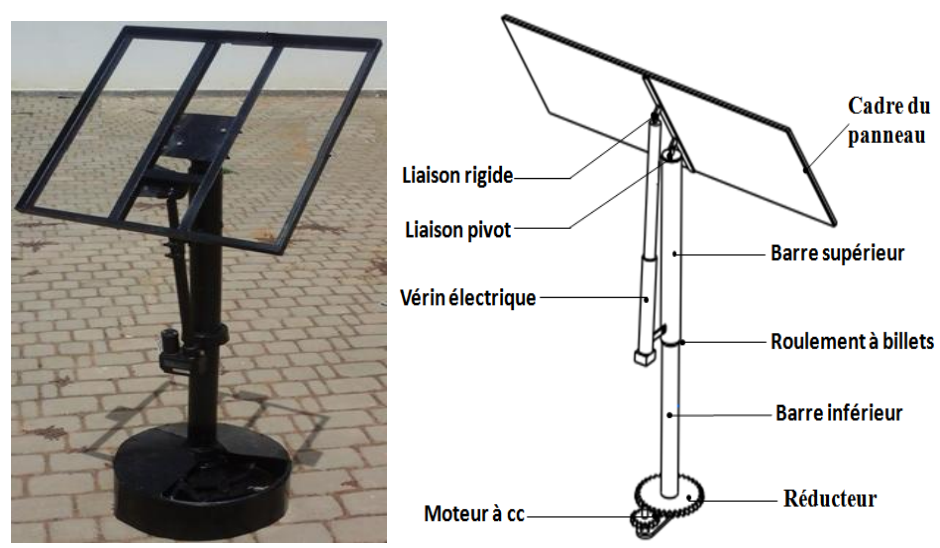

Fig. 4. Maquette du suiveur solaire

\section{Atelier2 : Le régulateur/chargeur de batterie}

Il s'agit de concevoir et réaliser un prototype de régulateur/chargeur de batterie au plomb $12 \mathrm{v} / 12 \mathrm{Ah}$ autonome, constitué d'un panneau PV connecté à un régulateur qui permet de charger la batterie (12v/12Ah). La conception de la régulation a pu se faire par recours à un circuit de régulation de tension qui permet, de fournir une tension continue de sortie $12 \mathrm{v}$ à partir d'une tension variable délivrée par un panneau. Quant à l'autonomie, un contrôle automatique de la batterie à l'aide d'un microcontrôleur connecté à un régulateur a pu être envisagé comme suit :

- le microcontrôleur déclenche la charge de la batterie à travers le régulateur de la tension de PV après analyse de la tension de l'entrée délivrée par le PV et de celle de la batterie,

- le microcontrôleur est alimenté directement par le PV à l'aide d'un autre régulateur de tension,

- le microcontrôleur assure l'affichage du niveau de tension de la charge de la batterie.

Ensuite, à l'aide du logiciel Proteus qui intègre ISIS, ils ont pu procéder à une simulation de la carte électronique inhérente au régulateur/chargeur de la batterie, simulation dont la réussite a conduit à la confection de la carte en question.

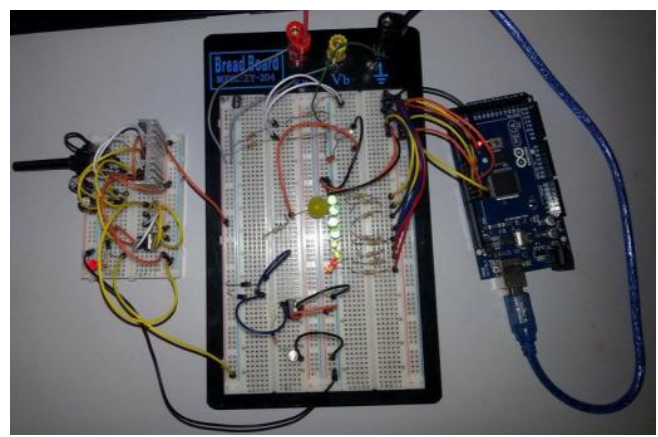

\section{Atelier3 : L'onduleur solaire}

Fig. 5. Carte du régulateur/chargeur de batterie

Il s'agit du produit de l'atelier 3 qui s'est intéressé à la mise en œuvre d'un prototype d'onduleur solaire avec une tension d'entrée de $12 \mathrm{VDC}$ et une sortie à onde purement sinusoïdale de $230 \mathrm{VAC}$ à une fréquence de $50 \mathrm{~Hz}$. La tâche n'a pas été facile d'autant plus que le produit attendu relève non seulement de l'électronique contenue dans le cursus normal de la formation universitaire mais aussi de l'électronique de puissance en particulier de l'utilisation de Mosfet's de puissance.

Dans un premier temps, les étudiants ont été invités de ce fait à étudier des documents en vue de les amener à glaner les éléments d'information nécessaires en matière d'électronique de puissance telle les différents interrupteurs de puissance de l'onduleur, la commutation, la consommation et la puissance transmise des Mosfet.

Dans un deuxième temps, ils se sont consacrés à l'élaboration d'une programmation de la carte Arduino Mega2560 qui permet d'assurer la commande et la surveillance de l'onduleur en question :

$\circ$ génération d'une onde purement sinusoïdale, 
- surveillance de la tension d'entrée,

- surveillance de la température des interrupteurs électroniques en vue de préserver le bon fonctionnement de l'onduleur (refroidissement avec des radiateurs et deux ventilateurs).

○ L'affichage de l'état de fonctionnement de l'onduleur

Dans un troisième temps, à l'aide deux logiciels ISIS et PSIM, ils ont pu procéder à une simulation de la carte électronique de l'onduleur, simulation dont la réussite les a conduits à la confirmation de l'efficacité du programme. Le quatrième temps a été consacré à la réalisation d'une carte de commande.

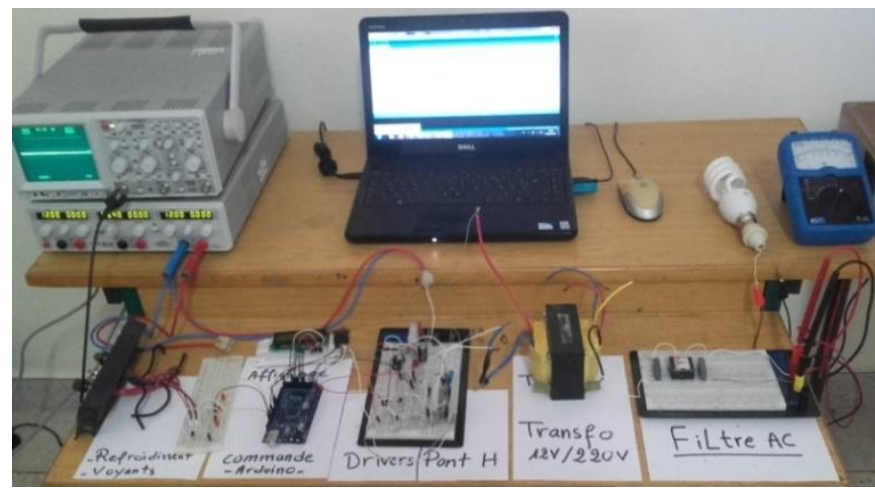

Fig. 6. Carte de l'onduleur solaire

Atelier4 : La carte automatique de la station de pompage d'eau

Il s'agit du produit de l'atelier 4 qui s'est intéressé à l'élaboration d'une maquette de station de pompage d'eau dont l'automatisation devait porter sur le remplissage d'un bassin, le déclenchement conditionné de la pompe immergée, la mesure du débit journalier de l'eau et le stockage instantané des informations sur PC (débit de la pompe, quantité d'eau stocké dans le bassin).

La nouveauté pour cet atelier a été dans le fait d'investir ses ressources cognitives de l'électronique dans un autre champ, celui du pompage de l'eau.

Dans un premier temps, les étudiants ont de ce fait été invités à étudier des documents Informatifs liés à la description, fonctionnement et dimensionnement de la station de pompage d'eau.

Dans un deuxième temps, ils se sont consacrés à la conception d'une carte de commande électronique qui permette l'automatisation du remplissage, du déclenchement, de la mesure et du stockage.

Dans un troisième temps, à l'aide du logiciel Proteus qui intègre ISIS, ils ont pu procéder à une simulation de la carte électronique de commande de la station, simulation dont la réussite les a conduits à la confirmation de l'efficacité du programme.

Dans un quatrième temps, dans une activité de dessin industriel soutenue par le logiciel CATIA, la conception de la maquette a pu connaître le jour, ce qui a facilité le passage à l'action, dans un cinquième temps consacré à la réalisation de la carte de commande et à la construction du prototype.

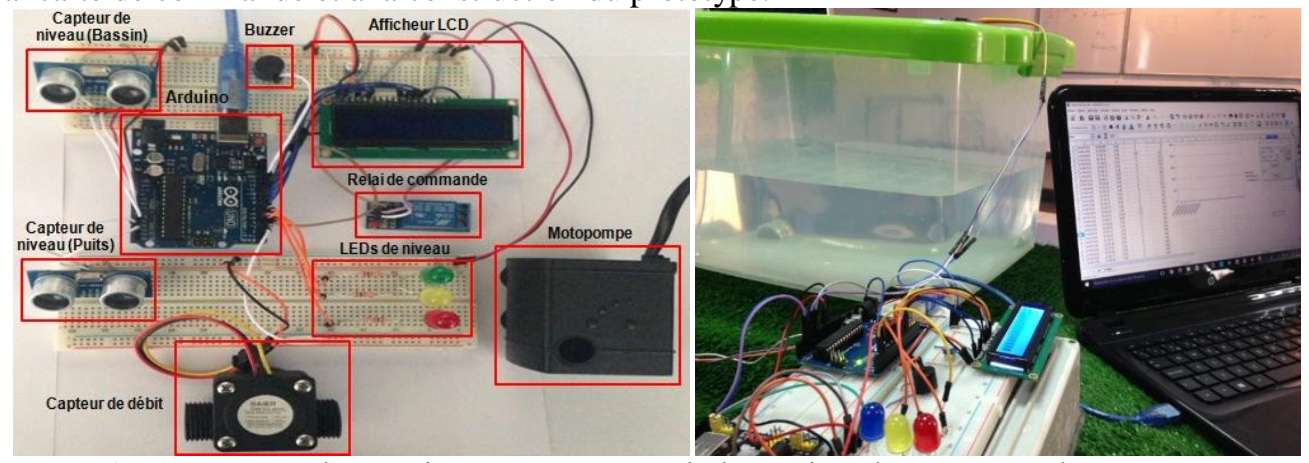

Fig. 7. Carte électronique et maquette de la station de pompage d'eau 


\section{Atelier5 : La carte de commande d'arrosage automatique}

Compte tenu des imperfections qualifiant les pratiques d'arrosage traditionnelles où la consommation de l'eau s'avère irrationnelle et $\mathrm{du}$ fait que les programmateurs en vigueur fonctionnent selon des horaires fixes préalablement enregistrés et partant, insoucieux des différences contextuelles inhérentes à l'humidité, la température, la ventilation et l'ensoleillement, l'atelier ${ }_{5}$ a vu nécessaire de développer une nouvelle modalité d'arrosage qui tient compte de la luminosité et de l'humidité du sol.

Le but a par conséquent été de concevoir et monter un prototype d'irrigation automatique à même d'assurer l'autonomie, l'intelligence et l'économie. Ce but a pu être concrétisé en amont par la conception d'un schéma où figurent une carte électronique, une pompe, un capteur d'humidité, un capteur de luminosité, une électrovanne et un buzzer.

Les étudiants ont été confrontés à une situation-problème qui se présentait comme suit :

Soit un bassin relié à une pompe surfacique reliée à un programmateur et faite pour refouler l'eau vers deux parcelles via une rampe à trois sorties qui se ferment et s'ouvrent à l'aide de deux vannes manuelles et une vanne automatique, les parcelles sont munies chacune d'un capteur d'humidité du sol, un buzzer connecté à un programmateur s'allume en cas de présence d'humidité dans le terrain1 à condition que le terrain2 est sec pour appeler à fermer les deux vannes manuelles, la vanne automatique est connectée au programmateur qui, selon l'humidité du terrain2, permet à cette vanne de s'ouvrir ou se fermer.

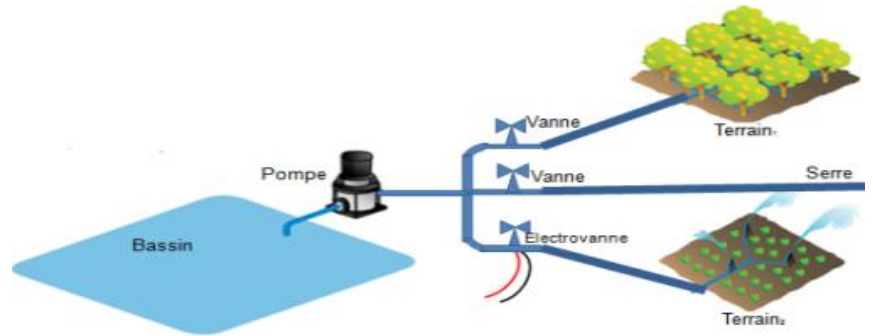

Fig. 8. Schéma d'arrosage

La tâche a été d'arriver à irriguer les deux parcelles en se servant exclusivement des composantes et données de la situation. En matière de luminosité, l'atelier a vu nécessaire de brancher le capteur de luminosité au programmateur de manière à ce que la pompe ne se déclenche que pendant la journée, lors de la présence du soleil, grâce au panneau photovoltaïque. Pour ce qui est de l'humidité, à l'issue d'une activité d'étalonnage du capteur d'humidité, quatre cas de figures se sont présentées :

Table 1 :- Table de vérité d'arrosage automatique

\begin{tabular}{|c|c|c|c|c|c|}
\hline \multirow{2}{*}{ Les cas } & \multicolumn{2}{|c|}{ Les terrains } & \multirow{2}{*}{ La pompe } & L'électrovanne & Le buzzer \\
\cline { 2 - 3 } & $\mathbf{( 1 )}$ & $(\mathbf{2})$ & & Terrain (2) & Terrain (1) \\
\hline $\mathbf{1}$ & humide & Humide & Off & Off & Off \\
\hline $\mathbf{2}$ & Sec & Sec & On & On & Off \\
\hline $\mathbf{3}$ & humide & Sec & On & On & On \\
\hline $\mathbf{4}$ & Sec & Humide & On & Off & off \\
\hline
\end{tabular}

L'état de la pompe (on / off), du sol (humide / sec) et de la luminosité sont d'autre part affichés automatiquement à un écran LCD

De plus, l'état du sol est visualisé à l'aide de trois LED selon les situations lisibles sur le tableau suivant :

Table 2 :- Table de vérité d'arrosage automatique

\begin{tabular}{|c|c|c|c|c|}
\hline Le terrain & L'humidité & La résistivité & Le LED & Irrigation \\
\hline SEC & $\mathrm{HMD}<25 \%$ & $>700$ & Rouge & Oui \\
\hline SEMI HUMIDE & $25 \%<\mathrm{HMD}<50 \%$ & $25 \%<\mathrm{RES}<50 \%$ & Jaune & Non \\
\hline HUMIDE & $\mathrm{HMD}>50 \%$ & $<400$ & Bleu & Non \\
\hline
\end{tabular}




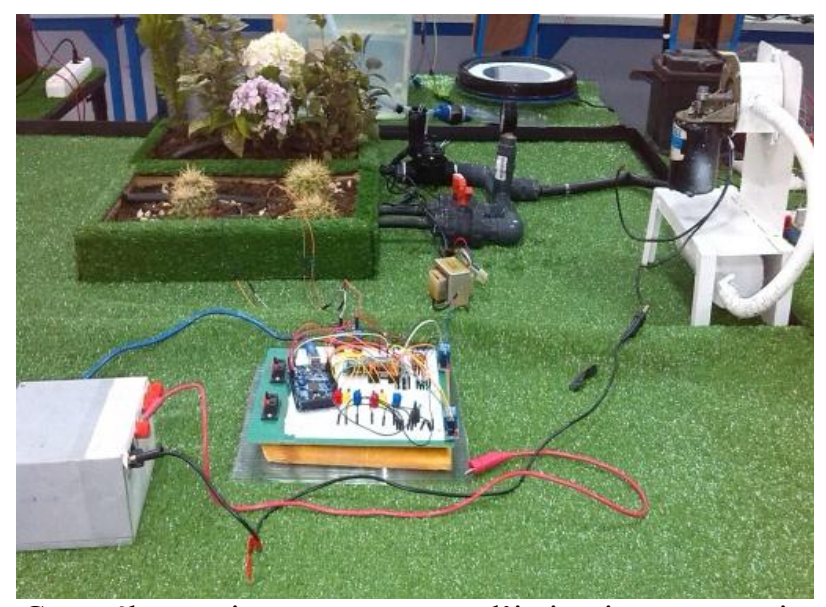

Fig. 9. Carte électronique et maquette d'irrigation automatique

Dans un deuxième temps, à l'aide du logiciel Proteus qui intègre ISIS, il a été possible de procéder à une simulation de la carte électronique d'irrigation automatique.

Dans une phase finale, une activité de dessin industriel soutenue par le logiciel CATIA a permis la conception de la maquette, ce qui a facilité le passage à la réalisation de la carte de commande et à la construction du prototype.

\section{Atelier6 : Le système de régulation de la température dans une serre photovoltaïque}

Etant donné la nature climatologique de la région du Nord, les cultures sont de plus en plus couvertes par les paysans en raison de leur crainte des effets de l'humidité et des importants écarts de températures entre la journée et la nuit. Or, ces couvertures, des serres de dimensions plus ou moins importantes, ne peuvent se prêter comme gage de protection des récoltes ni de leur épanouissement dans les meilleures conditions, d'où la nécessité de recourir à des techniques de régulation de la température par automatisation de l'affichage, de la ventilation, du chauffage et de l'ouverture / fermeture. C'est cette nécessité qui motiva le travail de l'atelier 6.

En matière de protection, avec un radiateur muni d'une carte de commande, il a été jugé possible de maintenir la température plus ou moins constante selon le type de culture.

En matière d'épanouissement, l'humidité a pu être dosée automatiquement à l'aide d'un brumisateur compresseur muni de carte de commande aussi et, avec un des capteurs d'humidité du sol munis toujours de cartes de commandes, la plante a pu avoir un dosage en eau compatible avec sa nature.

Le recours à l'automatisation a servi d'autre part à diminuer la chaleur due aux effets de serre et ce, par la mise en place d'un système d'ouverture automatique des fenêtres de la serre. Comme activités, il y eut un temps conception de carte électronique susceptible d'automatiser la ventilation, le chauffage et l'ouverture / fermeture des fenêtres de la serre.

Dans un deuxième temps, à l'aide du logiciel Proteus qui intègre ISIS, il a été possible procéder à une simulation de la carte électronique de commande de la serre.

Dans une phase finale, une activité de dessin industriel soutenue par le logiciel CATIA a permis la conception de la maquette, ce qui a facilité le passage à la réalisation de la carte de commande et à la construction du prototype. 


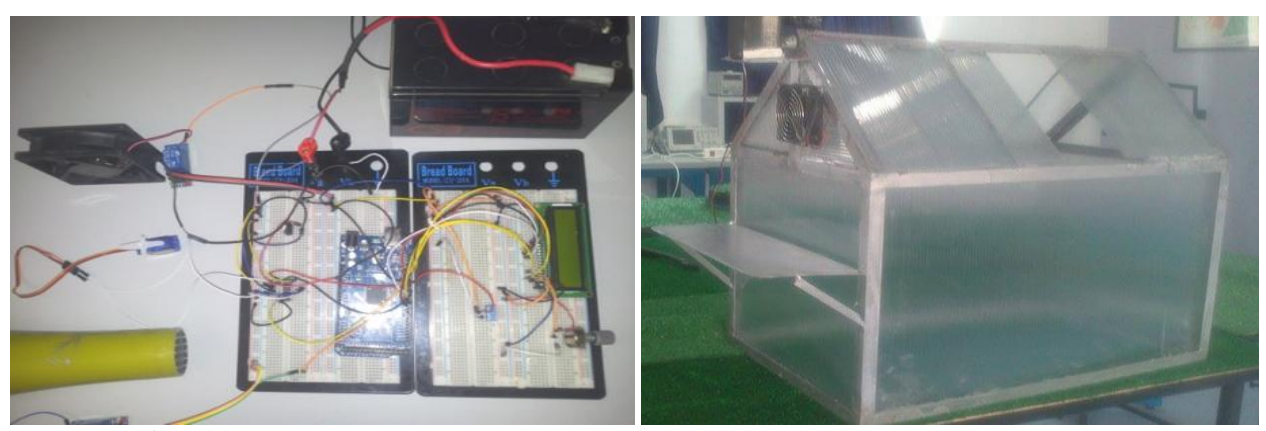

Fig. 10. Carte électronique et maquette de la serre automatisée

\section{Atelier7 : L'éclairage/surveillance automatiques du local de stockage des produits phytosanitaires}

Les produits phytosanitaires sont des préparations contenant une ou plusieurs substances actives. Ils servent :

- à protéger les plantes ou produits végétaux contre tout organisme nuisible,

- à exercer une action sur les processus vitaux des végétaux,

- à assurer la conservation des végétaux,

○ et à détruire les végétaux ou parties de végétaux indésirables.

Or, ces substances actives présentent à leur tour des dangers à l'emploi, raison pour laquelle elles sont ordinairement placées dans des armoires fermées à clef ou dans des locaux dont l'accès n'est pas libre.

Il s'agit en effet de produits qui craignent la chaleur, l'incendie et les personnes étrangères. Tous les cultivateurs sont conscients de ce fait et partant, leurs domaines agricoles ne sont guère conçus sans mesures de protection. C'est effectivement cette protection qui intrigua l'atelier7, lequel atelier s'est interrogé sur les moyens susceptibles d'assurer un maximum de sécurité automatique à la fois pour la ferme et pour la population. Comme activités, il y eut un temps de conception de carte électronique susceptible de déclencher automatiquement l'aération / ventilation pour réduire la température en cas de besoin, de déclencher subitement l'alarme en cas de tout soupçon d'incendie et d'empêcher toute intrusion de personne étrangère n'ayant pas antérieurement enregistré son empreinte digitale. Dans un deuxième temps, à l'aide du logiciel Proteus qui intègre ISIS, il a été possible procéder à une simulation de la carte électronique. Dans une phase finale, une activité de dessin industriel soutenue par le logiciel CATIA a permis la conception de la maquette, ce qui a facilité le passage à la réalisation de la carte de commande et à la construction du prototype.
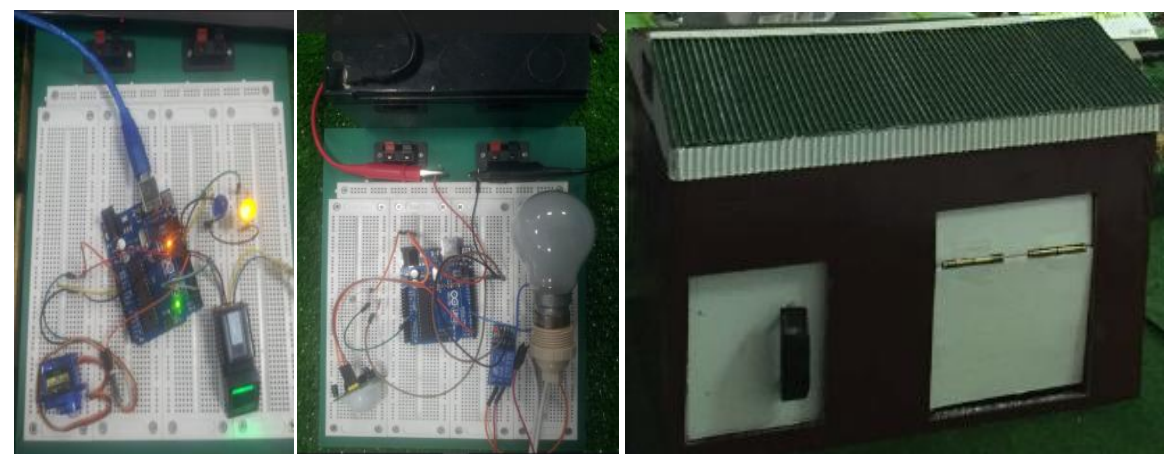

Fig. 11. Carte électronique et maquette d'éclairage automatique / Locale phytosanitaire

\section{L'APPORT COLLECTIF FINAL :-}

L'ensemble des apports groupaux ont pu enfin être réunis en une maquette où toutes les composantes se sont avérées fonctionnelles et interdépendantes. 


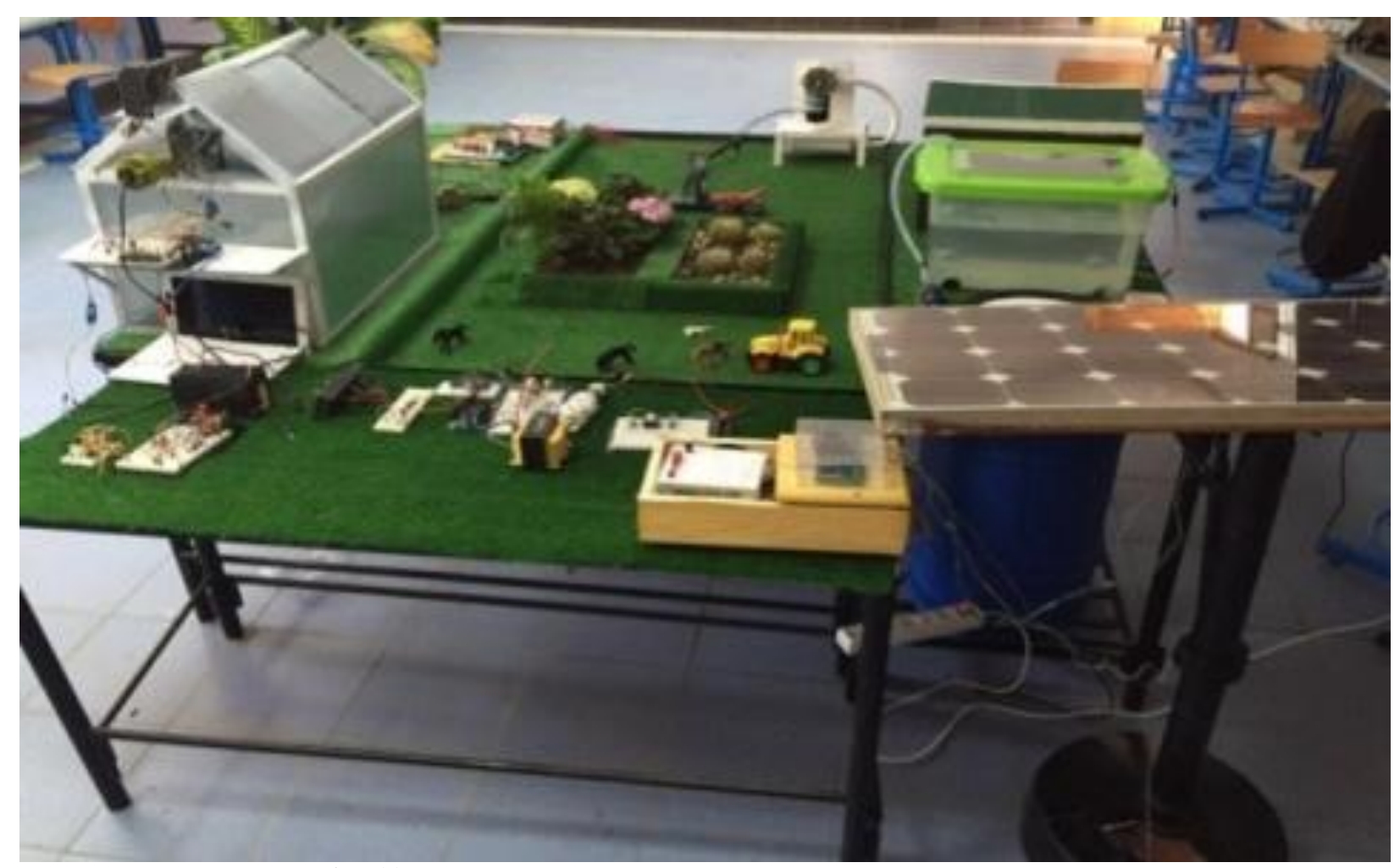

Fig. 12. Maquette du système Agricole à énergie renouvelable

\section{DISCUSSION :-}

La discussion a consisté en des séries d'évaluations puis en des perspectives d'extensions possibles, le tout ayant fait objet d'implication des étudiants, des professeurs et de personnes ressources invitées à cet effet.

\section{LES ACTIVITES D'EVALUATION :-}

L'EVALUATION DU PRODUIT :-

Une table ronde avec les étudiants a permis d'opérer les constats suivants : 
Table 3 :- Tableau récapitulatif et diagnostique du système Agricole

\begin{tabular}{|c|c|c|c|}
\hline \multirow{2}{*}{ Composantes } & \multirow{2}{*}{ Réalisations } & \multicolumn{2}{|c|}{ Appréciations } \\
\hline & & Positives & Négatives \\
\hline $\begin{array}{l}\text { Suiveur } \\
\text { solaire }\end{array}$ & $\begin{array}{l}\text { - Système mécanique bi axial et automatique } \\
\text { - Construction mécanique } \\
\text { - Réalisation de la carte de commande du prototype }\end{array}$ & \multirow{5}{*}{$\begin{array}{l}\text { - Fabrications } \\
\text { locales à } \\
\text { moindre coût } \\
\text { - Gestion } \\
\text { informatisée de } \\
\text { la station de } \\
\text { pompage } \\
\text { - Economie sur } \\
\text { les plans : } \\
\checkmark \text { énergétiqu } \\
\quad \text { e, } \\
\checkmark \text { pécunier, } \\
\checkmark \text { écologique }\end{array}$} & 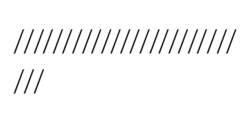 \\
\hline Onduleur & $\begin{array}{l}\text { - Tension de sortie purement sinusoïdale }(220 \text { volts, } 50 \mathrm{~Hz}) \\
\text { - Etat de fonctionnement affichable } \\
\text { - Refroidissement automatique à l'aide de radiateurs et } \\
\text { deux ventilateurs }\end{array}$ & & $\begin{array}{l}\text { Puissance } \\
\text { encore } \\
\text { insuffisante }\end{array}$ \\
\hline Régulateur & $\begin{array}{l}\text { - Fourniture de tension régulée de } 12 \mathrm{v} \text { à partir d'une } \\
\text { tension variable supérieure à } 6 \mathrm{v} \text { générée par le PV } \\
\text { - Contrôle automatique de la batterie via un } \\
\text { microcontrôleur connecté au régulateur et ce, à } \\
\text { travers les opérations suivantes : } \\
\checkmark \quad \text { analyse de la tension de l'entrée délivrée par le } \\
\text { PV et de celle de la batterie, } \\
\checkmark \quad \text { Auto-déclenchement de la charge de la batterie } \\
\checkmark \quad \text { Etat de fonctionnement affichable } \\
\end{array}$ & & $\begin{array}{l}\text { Régulation } \\
\text { encore } \\
\text { impossible des } \\
\text { tensions } \\
\text { inférieures à 6v } \\
\text { venant du PV } \\
\text { Augmenter } \\
\text { l'ampérage }\end{array}$ \\
\hline $\begin{array}{l}\text { Station de } \\
\text { pompage } \\
\text { solaire }\end{array}$ & $\begin{array}{l}\text { - Construction de la station de pompage d'eau } \\
\text { - Remplissage automatique du bassin } \\
\text { - Déclenchement conditionné de la pompe immergée, } \\
\text { - Mesure instantanée et stockage informatisé du débit } \\
\text { journalier de l'eau }\end{array}$ & & |/IIIIIIIIIIIIIIIIIIIII \\
\hline Irrigation & $\begin{array}{l}\text { - Construction d'une maquette d'arrosage automatique } \\
\text { - Développement d'une nouvelle modalité d'arrosage } \\
\text { en fonction de la luminosité et de l'humidité du sol }\end{array}$ & & $\begin{array}{l}\text { Capteurs } \\
\text { d'humidité du } \\
\text { sol à adapter à } \\
\text { la profondeur } \\
\text { selon la nature } \\
\text { du sol et la } \\
\text { culture et aux } \\
\text { besoins en eau } \\
\text { selon le type de } \\
\text { culture. }\end{array}$ \\
\hline Serre & $\begin{array}{l}\text { - Construction d'une maquette de serre } \\
\text { - Stabilisation automatique de la température } \\
\text { - Dosage automatique en humidité à l'aide d'un } \\
\text { brumisateur compresseur } \\
\text { - Déclenchement automatique de la ventilation, du } \\
\text { chauffage et de l'aération }\end{array}$ & $\begin{array}{l}\text { - Développement / } \\
\text { optimisation de } \\
\text { l'activité et de la } \\
\text { production }\end{array}$ & $\begin{array}{l}\text { Brumisateur à } \\
\text { perfectionner \& } \\
\text { Gestion de } \\
\text { risques : Prévoir } \\
\text { les ouvertures } \\
\text { manuelles }\end{array}$ \\
\hline $\begin{array}{l}\text { Locale de } \\
\text { stockage } \\
\text { phytosanitaire }\end{array}$ & $\begin{array}{l}\text { - Construction d'une maquette de local de stockage } \\
\text { - Stabilisation automatique de la température } \\
\text { - Déclenchement automatique de la ventilation/aération } \\
\text { - Déclenchement automatique de l'alarme en cas de } \\
\text { tout soupçon d'incendie ou d'intrusion }\end{array}$ & $\begin{array}{l}\text { agricole } \\
\text { - Contribution à la } \\
\text { protection de } \\
\text { l'environnement }\end{array}$ & |IIIIIIIIIIIIIIII \\
\hline Projet & $\begin{array}{l}\text { - Construction d'un système à composantes interactives } \\
\text { et complémentaires }\end{array}$ & $\begin{array}{l}\text { (eau, air, faune, } \\
\text { flore...) }\end{array}$ & $\begin{array}{l}\text { Besoin } \\
\text { d'installation } \\
\text { électrique/ } \\
\text { armoire de } \\
\text { distribution de } \\
\text { tension \& } \\
\text { Besoin de } \\
\text { supervision de } \\
\text { l'ensemble }\end{array}$ \\
\hline
\end{tabular}




\section{L'EVAluAtion DES COMPETENCES, CAPACITES, APTITUDES DES ETUDiANTS ET DES DEMARCHES ANDRAGOGIQUES :- Instrumentation de l'évaluation}

Nos instruments ont été variés en matière d'évaluation formative (Wiliam, 2010)(Scallon, 2004) pour laquelle il a été procédé à :

○ des prestations orales hebdomadaires de chaque atelier où l'appréciation était l'apanage non seulement du professeur mais aussi et surtout des autres ateliers dans une visée d'entrainement à la prise de parole, aux techniques de partage des idées et impressions, de développement de l'esprit critique, de l'esprit sportif, de l'esprit de responsabilité et de l'esprit de groupe, prestations qui ont du coup pu sensibiliser aux origines des erreurs / représentations erronées susceptibles de bloquer la poursuite du travail ultérieur et où les étudiants ont pu s'imprégner de techniques de défense du produit qu'ils auraient à valoriser,

- des rapports sur l'activité hebdomadaire de chaque atelier en alternance avec des tests pratiques devant le formateur,

- et des tables rondes mensuelles dans une optique de coordination et de régulation du débit de l'ensemble des ateliers.

En amont de l'évaluation sommative, les ateliers ont été invités chacun à exposer / commenter son produit final devant des personnes ressources en vue de bénéficier du regard étranger et de mieux s'enquérir des appréciations / orientations venant de domaines et secteurs multiples de l'activité et permettant de mieux répondre aux besoins tout en imaginant des extensions.

Pour des raisons certificatives, les soutenances ont eu lieu enfin devant un jury et un public qui n'ont pu qu'encourager les diverses prestations aussi bien que l'initiative de formation, abstraction faite du niveau de maîtrise de la langue française qui laissait encore à désirer.

La note finale de certification a pris en compte cependant non seulement la soutenance entreprise en fin d'année mais aussi les divers indices de productivité, de présence, de communication et ce, à travers les rapports intermédiaires, les tests pratiques, les prises de paroles, bref, les différents instruments d'évaluation utilisés le long de la session de formation consacrée au projet.

\section{Résultats des évaluations:-}

En matière de savoir, les étudiants ont été assez motivés pour dénicher l'information à travers les supports proposés aussi bien que par consultation de sites web, pour la traiter en fonction de leurs pré-acquis et pour l'investir dans les péripéties de conception / réalisation de leur projet, ce qui leur a permis non seulement de s'enquérir de connaissances mais aussi d'intérioriser celles-ci, de se les approprier comme étant leur découvertes, leurs biens.

En matière de savoir faire, les techniques d'animation adoptées ont permis le concours de tous les sens à travers des manipulations d'objets, des montages, des réfections, des erreurs et des essais qui ont du coup activé et réactivé leurs capacités d'observation, de comparaison, de sériation, d'analyse et d'extrapolation.

En matière de savoir être et devenir, les réussites réalisées à travers ces techniques inspirées des besoins de motivation, d'autonomisation et d'implication, qui se sont prêtés comme moyens de valorisation, ont servi de tremplins pour réaliser des gains de confiance en soi et aux autres avec lesquels tout le travail s'est poursuivi à travers les interactions et les adaptations, notamment en situations de prises de paroles devant des publics plus ou moins larges. Du coup, le sentiment d'avoir cherché et trouvé / inventé doit avoir sans doute doté les étudiants de souplesses suffisantes à l'entreprise de nouvelles tentatives de projets.

Néanmoins, par certains étudiants, la valorisation des produits n'a pu être satisfaisante à cause du niveau linguistique et culturel, une maîtrise peu réconfortante du français et une ignorance de l'évolution des projets auxquels il serait possible de comparer le leur pour l'apprécier à sa juste valeur. 


\section{LES EXTENSIONS :-}

Comme première tentative de formation à la lumière des approches par projets et nonobstant les multiples points positifs notés tant au niveau des étudiants qu'à celui du prototype réalisé, un certain nombre de détails reste à perfectionner, la science et le progrès étant toujours de natures inachevées.

\section{EXTENSIONS TECHNIQUES :-}

Comme extensions techniques, il serait possible d'augmenter la puissance fournie par l'onduleur pour couvrir tous les équipements électriques de la ferme, de confectionner un régulateur capable de stabiliser les tensions inférieures à $6 \mathrm{v}$ fournies par le PV, de mettre en place des brumisateurs au sein de la serre pour un bon dosage en humidité, d'utiliser et programmer des capteurs d'humidité de profondeurs variées selon la nature du sol, le type de culture et selon les besoins en eau qui dépendent des conditions climatiques ambiantes, de mettre en parallèle de tout système automatique un dispositif manuel de secours et de doter la ferme d'une armoire de distribution d e tension aussi bien que de logiciel de supervision de l'ensemble.

\section{EXTENSIONS GEOGRAPHIQUES ET INSTITUTIONNELLES :-}

L'initiative de formation aussi bien que le prototype élaboré par le biais de cette form'action auraient la chance de se faire valoir et de se perfectionner par leur transfert à d'autres villes, pays, centres de formation (facultés, écoles d'ingénieurs...) dans une visée de rayonnement implicatif de tout usager personnel ou institutionnel.

\section{EXTENSIONS DISCIPLINAIRES :-}

Tout d'abord et comme remarqué ci-dessus, les apports auraient connu plus de succès pour peu que la session de formation ait été antéposée et / ou accompagné de remises à niveau linguistique, de maîtrise d'un français suffisant pour approfondir les réflexions, assurer la fluidité de la communication et valoriser le produit réalisé.

Ensuite, pour une synergie plus fructueuse au sein de la faculté même, la contribution des tous les départements (mathématiques, informatique, biologie, économie, physique...) aurait pour mérite l'adoption d'une approche systémique de recherche-action éclairée par les finalités et les stratégies nationales de développement.

\section{EXTENSIONS PROFESSIONNELLES :-}

Comme nous avons focalisé cette année le travail sur les automatisations salubres d'un domaine agricole et compte tenu de la réussite de l'ensemble des actes réalisés, un éventail de perspectives s'ouvre en matière de transfert. Aussi nous serait-il possible de concevoir / réaliser ces mêmes automatisations dans les domaines des transports (véhicules à énergie solaire, motocycles pour les personnes aux besoins spécifiques...), de l'éducation-formation (production de matériel technique, recyclage de pièces encore intactes des équipements informatiques...), des services administratifs (confection de systèmes de surveillance / contrôle...), des industries (conception de moyens de réduction des émanations toxiques des usines...), etc.

\section{CONCLUSION :-}

Dans cet article, nous avons tenté de rendre compte d'un laborieux processus de productivité vécu "empathiquement" en compagnie des étudiants de la FPL de Larache, d'une implication collective dans un projet suggéré par une volonté de contribuer à l'amélioration de la vie par épuration de l'atmosphère et atténuation des dépenses, le projet DOSAER par le biais duquel nous avons pu améliorer la formation universitaire en lui octroyant un caractère innovationnel et significatif inspiré des théories d'apprentissage et des approches nouvelles liées à la conception / gestion de projets, à "l'exercitation " des capacités et le développement des compétences.

Dans une phase préopératoire, nous nous sommes consacrés au pré-requis / pré-acquis où nous avons élaboré un programme de formation préparatoire d'habilitation qui a énormément permis l'engagement des étudiants dans des exercices de recherches-actions menées en ateliers à tâches différenciées (Perrenoud, 2012) et complémentaires qui ont abouti en fin de compte à un modèle ô combien salutaire de réalisation.

Une phase postopératoire est venue ensuite donner l'opportunité d'évaluer les différents constituants de cette action andragogique, des produits des ateliers à la démarche méthodologique via les compétences des étudiants et la consistance du projet, le tout dans une visée de valorisation et d'incitation à la culture de la productivité. A travers, ces différentes évaluations, un certain nombre de perspectives d'avenir, traduites en extensions, se sont avérées passibles de mises en œuvre. 
Le projet DOSAER est on ne peut plus par nous considéré à la fois comme modèle technique dans la mesure où il permet l'adoption aisée des nouvelles technologies et comme modèle andragogique prometteur d'une série d'implications pluridisciplinaires à échelonner sur une série de projets de recherches inhérentes à diverses corporations.

Nous souhaitons en dernier lieu que notre initiative soit valorisée par son insertion parmi les actions et stratégies accrédités par l'Etat dans le cadre des politiques, stratégies et programmes salutaires dictées par l'impératif de généraliser l'usage de l'énergie solaire photovoltaïque et le besoin de toucher les zones plus ou moins éloignées du réseau électrique en particulier.

Nous souhaitons d'autre part que les différentes universités optent pour des projets de recherches-actions motivés par des commanditaires ministériels et / ou entrepreneuriaux selon des besoins régionaux réels collectés à travers des enquêtes permanentes et périodiques et ce, dans l'espoir de toujours lier la formation à la vie.

\section{REMERCIEMENTS :-}

Cette initiative n'aurait pas connu le jour sans la contribution en amont, en cours et en aval de personnes appartenant à des secteurs d'activités divers et dignes de grandes considérations pour leurs suggestions, leurs encouragements, leurs appuis multidimensionnel et leur présence permanente tant au niveau de la conception qu'à celui de la mise en œuvre et de l'évaluation.

A toutes ces personnes, il me sera à jamais un devoir de savoir gré tout en espérant les retrouver dans d'autres initiatives d'innovation et de développement en matière de Science et d'Education.

\section{Référence :-}

1. Vieillevoye, S., Wathelet, V. and Romainville M. (2012) : Maîtrise des pré-requis et réussite à l'université, In : M. Romainville et Ch. Michaut (dir.), Réussite, échec et abandon dans l'enseignement supérieur, Bruxelles : De Boeck. pp. 221-250.

2. P. Meirieu (1996) : Apprendre en groupe: Outils pour apprendre en groupe, Chronique sociale.

3. M. de Montmollin (1984) : L'intelligence de la tâche, berne, Peter Lang.

4. M. Pillonel, and J. Rouiller (2001): Faire appel à l'auto-évaluation pour développer l'autonomie de l'apprenant. Résonances, no. 7, pp. 28-31.

5. H. Charles (2012) : Comment impliquer l'élève dans ses apprentissages, ESJ Editeur.

6. X. Rogiers (2001) : Une pédagogie de l'intégration: compétences et intégration des acquis dans l'enseignement. DeBoeck Université.

7. N. Tardif (2005) : La pédagogie différenciée au service de l'apprentissage. Vie pédagogique, no. 134, pp. 2124.

8. Kahn, S. (2010): Pédagogie Différenciée. Bruxelles: De Boeck.

9. C. Baylon, X. Mignot (1994) : la communication. Paris, Nathan, 2e éd.

10. J. Proulx (2008): L’Apprentissage Par Projet, PUQ.

11. B. Galand, M. Frenay, (2005) : L'approche par problèmes et par projets dans l'enseignement supérieur, impact enjeux et défis, UCL Presses Univ de Louvain.

12. M.C. Presse, G. Figari, \& J-M. De Ketele, (2010) : La valorisation des expériences personnelles et professionnelles: introduction. Transformations, ${ }^{\circ} 4$, pp. $7-13$.

13. E. Bourgeois et J. Nizet, (1997) : Apprentissage et formation des adultes, PUF.

14. P. Perrenoud, (2012) : L'organisation du travail, clé de toute pédagogie différenciée. Issy-les Moulineaux: ESF éditeur.

15. Wiliam, D. (2010): Le rôle de l'évaluation formative dans les environnements d'apprentissage efficaces, In : H. Dumont, D. Istace and F. Benavides (dir.), Comment apprend-on ? La recherche au service de la pratique, Paris : OECD, pp. 143-170.

16. G. Scallon, (2004): L'évaluation des apprentissages dans une approche par compétences. Saint-Laurent : Éditions du renouveau pédagogique. 